\title{
Endoscopic Assisted Navigation Removal of Large Skull Base Wooden Penetrating Foreign Body
}

\author{
Amanda Almeida ${ }^{1}$, Eduardo Couto ${ }^{1}$, Laise Silveira ${ }^{1}$, and Carlos Chone $^{2}$ \\ ${ }^{1}$ UNICAMP \\ ${ }^{2}$ Universidade Estadual de Campinas Faculdade de Ciencias Medicas
}

January 5, 2021

\begin{abstract}
Penetrating orbitocranial trauma caused by foreign bodies requires prompt intervention. Nasal endoscopic approach is a less invasive technique. We describe an unusual case of a large skull base wooden penetrating foreign body submitted to this approach, in which a septal cartilage graft was used to reconstruct the medial orbit wall.
\end{abstract}

Endoscopic assisted navigation removal of large skull base wooden penetrating foreign body Amanda Sampaio Almeida ${ }^{1}$, Eduardo Vieira Couto $^{1}$, Laise Rodrigues Silveira ${ }^{2}$, Carlos Takahiro Chone ${ }^{1}$

${ }^{1}$ Department of Otolaryngology-Head and Neck Surgery, University of Campinas (UNICAMP), Vital Brasil St., 251, Cidade Universitária Zeferino Vaz. 13083-888. Campinas, SP - Brazil.

${ }^{2}$ Department of Radiology, University of Campinas (UNICAMP), Vital Brasil St., 251, Cidade Universitária Zeferino Vaz. 13083-888. Campinas, SP - Brazil.

Corresponding author: Carlos Takahiro Chone Department of Otolaryngology-Head and Neck Surgery, University of Campinas (UNICAMP), Vital Brasil St., 251, Cidade Universitária Zeferino Vaz; cchone@icloud.com

\begin{abstract}
Penetrating orbitocranial trauma caused by foreign bodies requires prompt intervention. Nasal endoscopic approach is a less invasive technique. We describe an unusual case of a large skull base wooden penetrating foreign body submitted to this approach, in which a septal cartilage graft was used to reconstruct the medial orbit wall.

\section{Key Words: Foreign Bodies; Intranasal Surgery; Facial Injuries; Or- bital Fractures}

Key Clinical Message: Management of injuries caused by penetrating skull base foreign body are complex and require a multidisciplinary care. Nasal endoscopic approach preserves the functionality of the structures in a less invasive technique.

\section{Introduction}


Penetrating orbitocranial trauma caused by foreign bodies is related to high impact trauma and imposes severe risks of critical structure damages, as meningitis, vision loss, fistula and death ${ }^{1}$. Besides, it is often associated with other facial injuries and therefore, demand multidisciplinary care ${ }^{2}$. A prompt intervention is recommended in cases associated with organic foreign body, such as wood, due to the risk of infection ${ }^{3}$.

Preserving the functionality of the structures, in addition to restoring aesthetic characteristics, are the main objectives of surgical treatment. In this scenario, new surgical techniques have been described, highlighting the use of endoscope and a transnasal approach ${ }^{4}$. Due to the rarity of skull base foreign bodies, it is interesting to report the cases, as may contribute to the decision of the surgical repair and further create a treatment algorithm. Here we present an unusual case of large skull base wooden penetrating foreign body submitted to endoscopic sinus surgery in an emergency setting.

\section{Case Report}

A 29-year-old man admitted to the emergency room with a history of being beaten with a tree branch that transfixed his head through the inferolateral region of the left orbit (Figure 1). He had been previously intubated and sedated at a primary hospital due to decreased consciousness (Glasgow Coma Scale: 7/15). His medical historical was unknown, except for drugs abuse.

After initial management provided by trauma surgeons, the patient was submitted to full body computed tomography (CT). CT scans showed a foreign body with approximately fifteen centimeters penetrating skull base through his left orbit region, with inferior and posterior way to the right temporal muscle. Several comminuted fractures were seen as: medial and inferior walls of left orbit, left lamina papyracea, nasal septum, medial and lateral walls of right maxillary sinus (Figure 2). Nasal bone was bilaterally fractured. Furthermore, foci of subarachnoid hemorrhage were also detected (Marshall 2).

Multidisciplinary evaluation was conducted by neurosurgery and ophthalmology. As the ocular globe seemed preserved and no significant brain injury was detected, a conservative approach was adopted by those specialties. Patient was then transferred to operation room by the otolaryngology team. First, hemorrhage from the right maxillary artery was controlled under endoscopic visualization. After, we performed a careful manual removal of the foreign body (Figure 3). After extenuating nasal irrigation and removal of wood pieces, nosebleeds were controlled with adrenaline solution. As the orbital fat was prolapsing into the nasal cavity, grafting of the medial orbital wall was performed with a septal cartilage flap (Figure 4). The patient remained in intensive care and empirical antibiotic therapy was started with third generation cephalosporin and clindamycin. Tetanus vaccine was also administered. In the postoperative ophthalmological evaluation, the left eye was de-epithelialized, but due to agitation, he remained sedated, with extubation failures and, therefore, a complete evaluation was not possible.

Ten days after procedure, patient still presented fever despite broad-spectrum antibiotic and negative cultures, therefore, a second emergency endoscopic nasal surgery was performed to drainage hemossinus. On fifth postoperative-day, he developed acute kidney injury, hypernatremia, and died after an irreversible cardiorespiratory arrest.

\section{Discussion}

Considering the severity of the trauma, the initial management should follow advanced trauma life support systematization. A multidisciplinary assessment should be followed, including neurosurgery and ophthalmology, and foreign body removal is postpone until clinical stabilization is guaranteed ${ }^{5}$.

It is imperative to perform an image exam to assess the extent of the damage, so a computed tomography scan (CT) is essential. In the scenario of traumatic brain injury, CT allows a fast assessment, with good tomographic-clinical correlation ${ }^{6}$. In addition, it is the best modality to evaluate bone deformities and has good sensitivity to foreign bodies, except for the detection of wood particles ${ }^{7}$. According to a review of twenty-four case reports, wooden foreign bodies can be me missed in almost one third of initial exams as it can be confused as free air ${ }^{8}$. In our case, despite the organic composition of the object, CT contributed 
to therapeutic planning, as it surprisingly did not show any significant intracranial damage or to the ocular globe, therefore, it was decided to remove the foreign body.

Surgical approach depends on the degree of involvement and nature of foreign body. Regarding the material, orbital organic foreign bodies should be removed within twelve hours due to the risk of infection ${ }^{9}$. In cases where there is no impairment of the orbit or vascular structures, the foreign body can be removed with immediate orbital repair ${ }^{2,3}$. The literature presents two main foreign body removal techniques: external access or by transnasal endoscopy ${ }^{10}$. External access has the advantage of better control of hemorrhages and direct visualization of the foreign body and surrounding injured tissues ${ }^{10}$. The endoscopic access of the skull base was highlighted for being a less invasive treatment, with less morbidity and greater aesthetic gain, in addition to allowing the repair of fistulas at the same surgical time ${ }^{11}$. However, there is a lack of prospective studies that compare the superiority of the different accesses mainly because of the rarity of foreign body cases in skull base. Therefore, the choice will depend on the extent of the damage and the surgeon's experience ${ }^{12}$.

Regarding postoperative care, immunization against tetanus is mandatory according to the vaccination situation ${ }^{13}$. In addition, despite the lack of evidence, several case reports support the use of prophylactic

antibiotic therapy to prevent complications such as abscesses and meningitis ${ }^{14}$. The scheme used in the literature varies, and there is no consensus about the type, timing or duration ${ }^{15}$.

In our patient, it was possible to remove the foreign body manually. We used the endoscopic technique to control nasal bleeding, remove retained fragments and to reconstruct the papyraceous lamina, which was possible without a metal plate. The correction of other fractures was postponed until the edema and clinical conditions improve.

\section{Conclusion}

Foreign bodies in the skull base are rare and can cause severe damage and complications, such as meningitis, vision loss, fistula and death. Initial management should follow the principles of advanced trauma life support. After stabilization, it is necessary to assess the integrity of the structures, so multidisciplinary care and a computed tomography are essential. We recommend the removal of the foreign body in an operating room environment and the simultaneous use of sinus endoscope to ensure bleeding control and repair lesions under direct visualization with a less invasive approach.

Acknowlodgments: None.

Conflict of interest: None declared.

Author Contributions: AA and EC participated in the surgical management of this case and were involved in the study's design and writing. LS contributed in radiological assessment and data analysis. CC contributed with literature review and manuscript edition. All authors read and approved the final manuscript.

\section{References}

1. Klančnik M, Ivanišević P, Lupi-Ferandin S, Sučić A, Ledenko V, Lešin M, et al. Penetrating orbitocranial injury. Acta Clin Croat. 2018;57(4):792-6.

2. Martín Fernández-Ferro MF, , Jacinto Fernandez-Sanroman, Alberto Costas-Lopez AL. Management of a Penetrating Orbital Trauma from an Unusual Foreign Body with Associated Eye Injury. Ann Maxillofac Surg. 2019;9(1):214-7.

3. Barouj MD, Tabrizi R, Behnia P, Amir M, Tabrizi A. Penetrating Orbital Injury ; a Case Report and Treatment Algorithm. 2020;8(1):3-7.

4. Pham AM, Strong EB. Endoscopic management of facial fractures. Curr Opin Otolaryngol Head Neck Surg. 2006;14(4):234-41. 
5. Santos T de S, Melo AR, Moraes HHA de, Almeida Junior P, Dourado E. Impacted foreign bodies in orbital region: review of nine cases. Arq Bras Oftalmol. 2010;73(5):438-42.

6. Gasparetto EL. Tomografia computadorizada no traumatismo cranioencefalico. Radiol Bras. 2011;44(2):3984.

7. Moretti A, Laus M, Crescenzi D, Croce A. Peri-orbital foreign body: A case report. J Med Case Rep. $2012 ; 6: 2-5$.

8. Jusue-Torres I, Burks SS, Levine CG, Bhatia RG, Casiano R, Bullock R. Wooden Foreign Body in the Skull Base: How Did We Miss It? World Neurosurg. 2016;92:580.e5-580.e9.

9. Shyam Sundar Das Mohapatra JDHB. An unusual case of orbitocranial wooden foreign body with amazing outcome: A case report. Indian J ophthalmol. 2020;68(1):219-21.

10. Tsao Y, Kao C, Wang H, Chin S, Moe KS, Diego S. Transorbital Penetrating Injury of Paranasal Sinuses and Anterior Skull Base by a Plastic Chair Glide : CASE REPORT. 2006;(325):177-9.

11. Presutti L, Marchioni D, Trani M, Ghidini A. Endoscopic Removal of Ethmoido-Sphenoidal Foreign Body with Intracranial Extension. 2006;244-6.

12. S Thomas, A Daudia NSJ. Endoscopic removal of foreign body from the anterior cranial fossa. 2007;121:794-5.

13. Yoshihara S, Baba S, Kanemaru A, Ichikawa T. Craniofacial penetration by a wooden stick. Eur Ann Otorhinolaryngol Head Neck Dis [Internet]. 2019;136(5):393-5.

14. Wen YH, Hou WJ, Lei W Bin, Chen FH, Zhu XL, Wang ZF, et al. Clinical Characteristics and Endoscopic Endonasal Removal of Foreign Bodies within Sinuses, Orbit, and Skull Base. 2017;130(15).

15. Zhang D, Chen J, Han K, Yu M, Hou L. Management of Penetrating Skull Base Injury : A Single Institutional Experience and Review of the Literature. 2017;2017.

FIGURE LEGENDS

Figure 1. Preoperative status.

Figure 2. Preoperative computed tomography

Figure 3. A) Removal of the foreign body; B) The wooden fragment.

Figure 4. Endoscopic view.

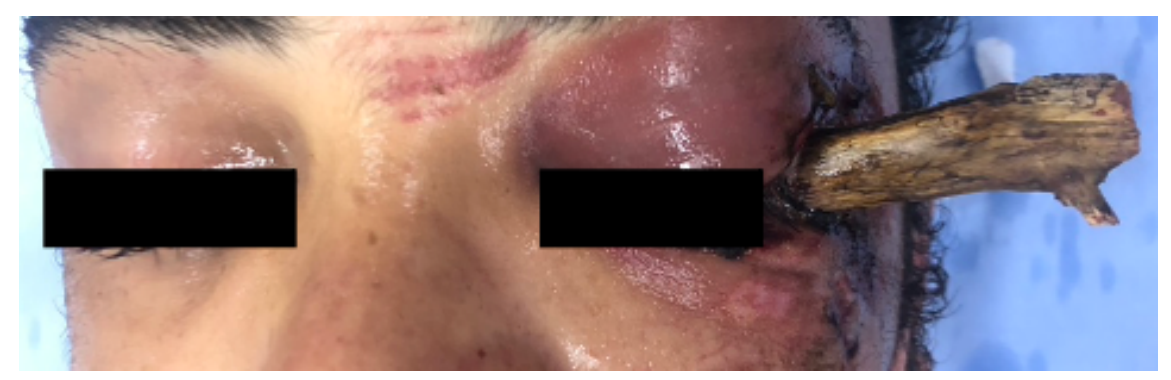



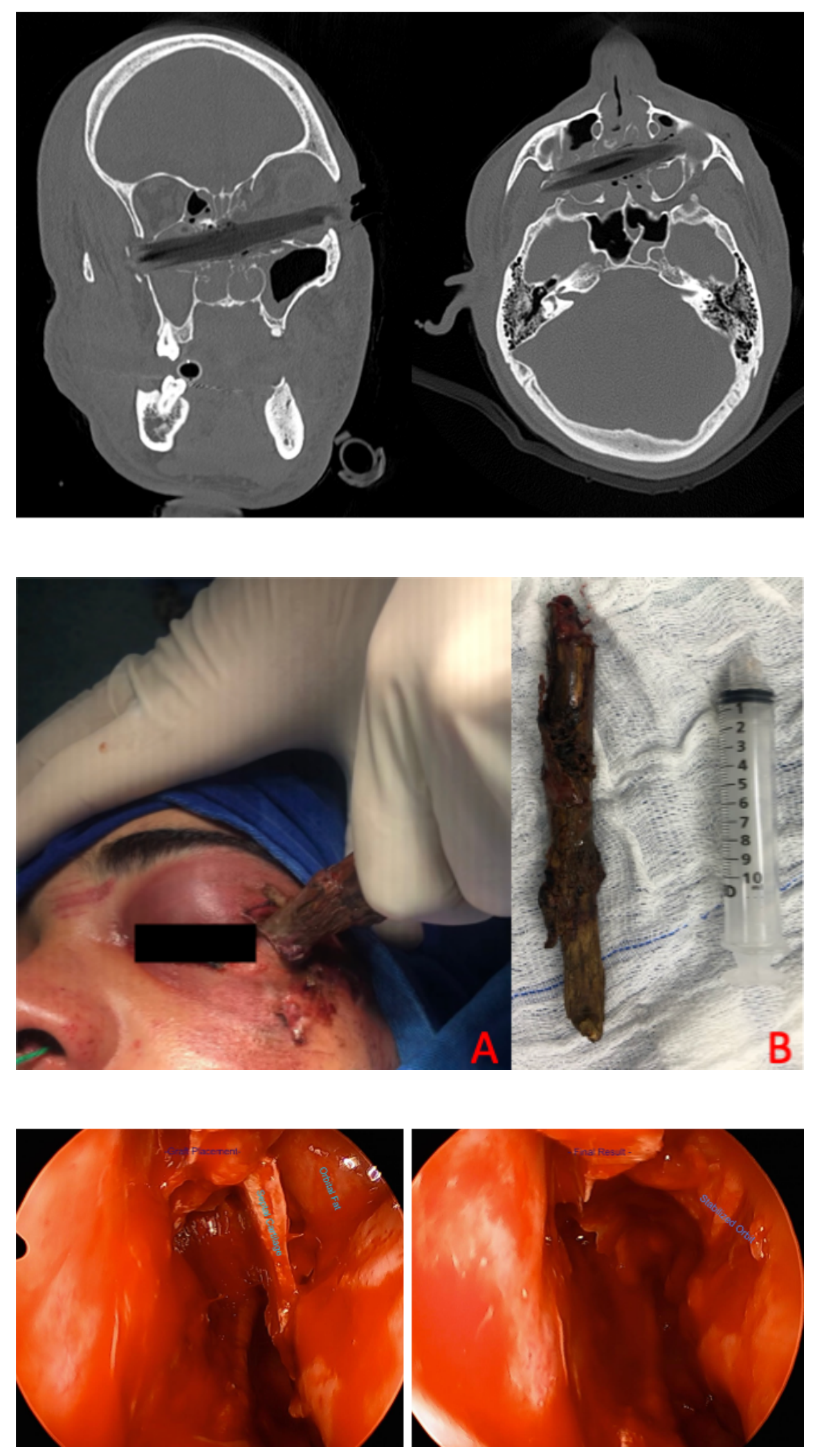